\title{
El castillo de Almansa: ejemplo de adaptación de un castillo a las teorías de la fortificación del siglo XV \\ Joaquín Francisco García Sáez
}

Instituto de Estudios Albacetenses “Don Juan Manuel”, Albacete, España, eachimo@ymail.com

\begin{abstract}
The Castle of Almansa has its origin in a primitive Islamic fortress remodeled by Don Juan Manuel, which is the fortress that will inherit Don Juan Pacheco, who as Marquis of Villena will transform, adapting to new theories of fortification that arise in the fifteenth century mainly derived from the widespread use of pirobalística artillery. This adaptation of Don Juan Manuel's obsolete fortress, becomes Don Juan Pacheco's castle in an example of the theories of the fortification of the fifteenth century, with the addition of several elements depending on the one hand, the characteristics of the castle was found, and secondly, the geological nature of the hill and its relationship with the surrounding environment, while made him the image of the expression of the power that a character like Don Juan Pacheco once had.
\end{abstract}

Keywords: Fortress, example, fifteenth century, Pacheco.

\section{El Castillo de los Pacheco. Siglo XV}

El Castillo de Almansa es una fortificación, emplazada en el cerro del Águila que se ha ido conformando a lo largo del tiempo, en un proceso que se inició la época almohade, y que se culmina en el siglo XV con las actuaciones que Juan Pacheco y su hijo Diego López Pacheco realizan para adaptar la fortaleza que heredan a las técnicas de la fortificación de ese momento.

A pesar del estado de ruina que nos ha llegado este castillo, agravado por las restauraciones sin rigor histórico que se realizaron en el siglo XX, se han conseguido identificar, gracias a las últimas investigaciones historiográficas como pudieron haber sido los modelos del castillo de Almansa del siglo XIV, que perteneció y construyó Don Juan Manuel y el de los Pacheco del siglo XV.

El castillo que encuentra el Marqués de Villena, es un castillo anticuado que responde a las necesidades defensivas del siglo XIV, por lo que el castillo se podría definir como inútil para el papel de una fortaleza en los asedios del siglo XV.

Situado en un enclave estratégico, como es el Corredor de Almansa, en tierra fronteriza entre los reinos de Castilla y Aragón, urge adecuarlo a los nuevos tiempos, adaptándolo a las teorías de la poliorcética y de la fortificación del momento, cuya principal novedad es la introducción de la artillería pirobalística, tanto para defender como para atacar la fortaleza, además de controlar sus puntos débiles y reforzarlos. Para eso Don Juan Pacheco contrataría a los mejores ingenieros del momento, probablemente de la vecina Valencia (Martínez, 2015).

Pero estas actuaciones, además de adaptar la edificación a las teorías de la fortificación de los nuevos tiempos para que el castillo se comportase como un edificio inexpugnable, tendrán otra misión tan importante o más que ésta, que sería la de manifestar el poder de su 
propietario que, como es bien sabido en el caso de Don Juan Pacheco, rivalizaba con el de los propios reyes de Castilla (Villena 2000).

Es por esto que un castillo en esta época, no solo debería ser inexpugnable, sino manifestar explícitamente esa inexpugnabilidad como parte de esa manifestación del poder de su propietario.

Todos los castillos están condicionados por su emplazamiento, condición que los hace únicos a todos y a cada uno de ellos, y el de Almansa no será una excepción.

Partiendo del conocimiento del entorno inmediato, empezando por el conocimiento del propio cerro del Águila, se plantean las correspondientes soluciones para hacer al Castillo de Almansa heredado de los tiempos de Don Juan Manuel, una fortaleza inexpugnable a los asaltos del siglo XV, para lo cual se realizarán las actuaciones respondiendo al edificio heredado, a la heterogeneidad del entorno y, por supuesto, a la del cerro.

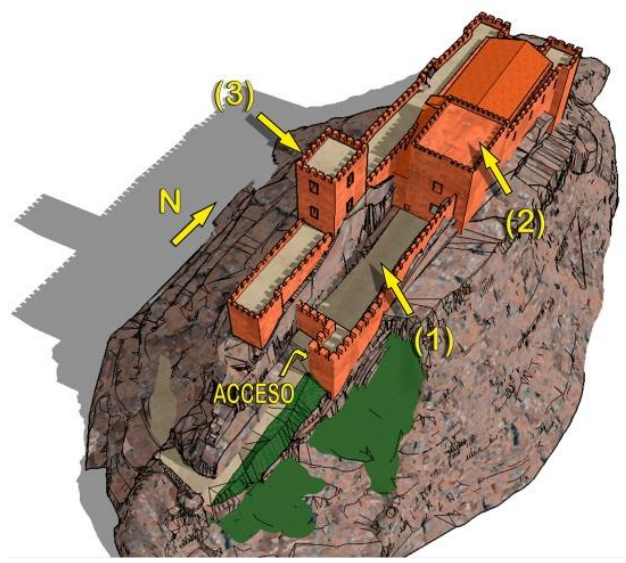

Fig. 1- Hipotesis del Castillo de D. Juan Manuel (Joaquín Fco. García, 2015)

Relativo al castillo heredado, el edificio que se encuentra Don Juan Pacheco, es un recinto amurallado de planta aproximadamente rectangular, adaptado a la forma del cerro, y por tanto la dimensión mayor del rectángulo estará dispuesta según la dirección norte-sur, con torres en sus cuatro vértices unidos por lienzos de muralla construídos con la técnica del tapial y apoyados sobre las lajas de roca verticales del cerro, de manera que estos lienzos, en su base son la propia roca del cerro.

El único acceso a la fortaleza está en la fachada sur.

En su interior tres zonas: el patio de armas junto al acceso (1), dependencias del castillo junto al patio (2) y la torre del homenaje (3) situada en la parte alta del Castillo, a modo de último reducto con aljibe propio.

El Castillo está orientado de forma tal que su dimensión mayor sigue aproximadamente la dirección de un eje norte-sur.

Observando el entorno del castillo, se constata que su lado oeste es la zona orográficamente más complicada, mientras que tanto las fachadas norte, este y sur se abren al gran espacio, a modo de llanura conocido como el Corredor de Almansa.

La población de Almansa se sitúa en su lado oeste, protegida por la orografía y el Castillo.

Esto implica que la aproximación al castillo va a ser más fácil por cualquiera de estas fachadas que por la oeste.

Pero a su vez, como ya se ha comentado, tanto el castillo, como el cerro del Águila presentan características distintas por cada una de sus orientaciones por lo que la respuesta que se da para la adaptación de la fortaleza a los tiempos del siglo XV va a plantearse de forma diferente en cada una de ellas.

\subsection{Fachada Sur}

En esta fachada, dos son los puntos débiles que se encuentran que afecten exclusivamente a ella.

El primero, y derivado del propio funcionamiento de la edificación, sería el acceso, ya que todo acceso en un recinto amurallado se considera un punto débil de la fortaleza, aunque sea inevitable, puesto que por un punto hay que acceder a ella.

El segundo es el propio cerro, ya que el lienzo de la puerta está construido sobre un estrato blando del cerro, y por tanto fácil de excavar. 


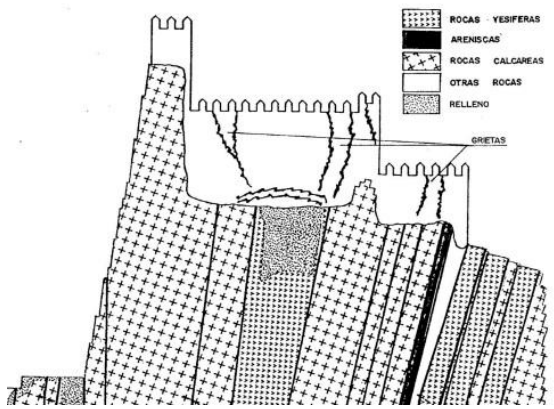

Fig. 2- Sección oeste-este de los estratos verticales del cerro del Águila (Ángel Uriel, 1990)

Además al castillo habrá que reforzarlo para resistir los embates de la nueva artillería pirobalística, por lo que son tres las actuaciones que se realizan en esta fachada son para solucionar estas "deficiencias" adaptando al castillo a las nuevas teorías de la fortificación del siglo XV.

La primera actuación tiene que ver con el acceso. Como se ha comentado, la entrada no se puede eliminar, pero si reforzar su defensa.

En este caso, interponiendo otras construcciones a modo de barreras que hagan más difícil el acceso, para lo cual se construye una barbacana con acceso en "codo", en cuyo portón, además, se dispone una buhedera para incrementar la primera defensa.

La segunda actuación viene derivada de la naturaleza del cerro.

Relativo al problema del cerro de presentar un estrato fácilmente excavable en esta zona, desde el punto de vista poliorcético, implica que el castillo es susceptible de ser atacado por la técnica de la zapa o mina.

Tradicionalmente la respuesta que se plantea desde el punto de vista de la fortificación para evitar este tipo de ataques es la construcción de un foso, sea seco o inundable.

Así pues para solucionar este segundo problema, en el castillo de Almansa se construye un foso seco delante del lienzo sur del castillo de Don Juan Manuel, y por tanto también delante de la puerta acceso al castillo.
La generación de este foso hace desaparecer la continuidad física del camino que discurre por la barbacana hasta el acceso a la fortaleza propiamente dicha, lo que hará necesario la realización de un puente levadizo o pasarelas deslizantes que permitan el paso desde la barbacana al interior del recinto fortificado.

De esta manera, además de protegerse frente a la zapa, se incrementa la defensa del acceso del Castillo interponiendo una barrera más para el acceso.

Con las tierras procedentes de la excavación del foso se realizarían parte las construcciones de tapial del ala sur del Castillo, concretamente las que se han documentado que se realizaron en el siglo XV (Gil, 2008) y el refuerzo de la fachada norte en forma de baluarte.

Utilizando así todos los recursos del propio cerro, haciendo de un material de desecho un material útil para la reforma y refuerzo de la fortaleza (García, 2011).

Pero no se trata de un foso corrido en toda la dimensión de las fachadas, como pudiera serlo el de Chinchilla.

El foso del castillo de Almansa, está situado solo en la fachada sur, acotado entre dos estratos verticales de naturaleza caliza que lo delimitan por el oeste y el este, sólo donde el terreno es deficiente, pero este foso, situado en el extremo sur del cerro no tiene una contraescarpa de forma natural ya que la obra para realizar el foso en el castillo de Almansa, no sería un vaciado para la ejecución de una zanja, de mayor o menor dimensión, como en Chinchilla, sino un desmonte de terreno blando entre estos dos estratos verticales calizos de gran dureza.

Al realizar una zanja, se generan dos paramentos verticales, la escarpa junto a la muralla y la contraescarpa enfrente que provocan que, para acercarte a la fortaleza, haya que saltar el foso, pero en el caso del desmonte, si bien es cierto que aumentas la altura del paramento defensivo, se podría llegar hasta este paramento fácilmente y su ataque sería sencillo.

Para poner trabas a esta situación, y por tanto mejorar la defensa de esta fachada, 
especialmente sensible por estar en ella el acceso al recinto fortificado principal, lo que se hace es construir una "contraescarpa" o limitación exterior del foso, para cerrar este vacío y recuperar el concepto de zanja, dificultando el acercamiento al paño de la fortaleza donde se sitúa el acceso.

Así este foso sería un vacío de planta aproximadamente rectangular situado en el extremo sur del cerro, limitado por su lado norte por la fortaleza, por su lado oeste por el estrato rocoso vertical de naturaleza caliza sobre el que se desarrolla la barbacana y, por su lado este, por un estrato de la misma naturaleza, pero más estrecho, coronado con un muro de fábrica de mampostería para hacerlo más inaccesible, y en el lado sur, para cerrar este espacio se construye una torre albarrana adosada a la barbacana a través de la cual se accedería, con el fin, de situarla justo delante del paño de la entrada, a modo de torre albarrana de flanqueo (De MoraFigueroa, 2006).

Se genera así un mecanismo defensivo compuesto de barbacana y foso, limitado por la torre albarrana.

Todas estas construcciones están constatadas que son del siglo XV, porque en ellas aparecen los escudos de los Pacheco, se construyen con mampostería y se revisten con un enlucido de mortero de cal acabado con dibujos de recercados de formas ovoides que se repiten en todos los castillos del Marqués de Villena, desde Sax a Castillo de Garcimuñoz, pasando por Villena, Jumilla, Chinchilla, Alcalá del Júcar o Belmonte.

Por último, la tercera actuación, relativa al refuerzo global del castillo para adaptarlo a la defensa de la artillería pirobalística, en esta fachada en su lado oeste, en el extremo de lo que se conoce como ala sur del Castillo, se construye un recrecido macizo de planta semicircular adosado a la construcción extrema de tapial cuyo única finalidad es la de reforzar ese paramento de tapial, para que el impacto de los proyectiles que se lanzaran fueran lo menos destructivos posible, ya que el impacto sobre un paño de directriz curva es menos agresivo que sobre un paño cuya directriz sea recta.
Al día de la fecha solo aparece este refuerzo en la parte oeste de la fachada sur, pero no tiene sentido que si se refuerza la "esquina" oeste, no se haga lo mismo con la este, en la que además en la parte este de la fachada está situado el acceso a la fortaleza, razón de más para reforzarla.

Investigaciones historiográficas confirman la existencia de este refuerzo (García, 2015) y actuaciones en otros castillos como el de Biar, que también refuerza la esquina del acceso, lo refrendan.

Así pues la fachada sur del recinto fortificado del castillo de Almansa tendría dos refuerzos de planta circular realizados en el siglo $\mathrm{XV}$, en pos de su adaptación a los nuevos tiempos, el suroeste que ha llegado hasta nuestros días y el sureste que ha desaparecido.

\subsection{Fachada este}

Esta fachada se sitúa sobre un estrato rocoso vertical de naturaleza caliza, y hacia el este o, lo que es lo mismo, hacia el exterior de la fortaleza se disponen otros estratos verticales también de naturaleza caliza y/o yesífera de menor altura formando una suave pendiente descendente en el perfil del cerro, alejándose de la fortaleza, que se anclan en el terreno a muchos metros de profundidad.

La profundidad y la dureza de estos estratos hace prácticamente inviable el ataque por mina, por lo que la realización de un foso es totalmente innecesario, hecho por lo que no se extiende por esta fachada y queda limitado a la fachada sur.

Pero debido a la relativa suavidad de la pendiente del cerro en esta orientación hace a esta fachada relativamente accesible y los muros que la defienden son de tapial, relativamente débiles al ataque pirobalístico.

Además esta fachada está orientada a la llanura conocida como corredor de Almansa que permite el establecimiento de baterías de artillería para poder atacar al castillo desde este punto.

Así pues el refuerzo del castillo que se propone para esta fachada es la construcción de una 
falsabraga (De Mora-Figueroa, 2006) o acítara artillada con el que poder hacer frente a los posibles ataques que por esta orientación pudieran venir.

En esta falsabraga aparecen troneras con palo y orbe.

Se trata de colocar una barrera para impedir el acceso directo a los lienzos de la fortaleza propiamente dicha, evitando su posible ataque por escala, y además situar una línea defensiva de artillería delante del recinto fortificado principal para defenderse de los ataques que pudieran venir por esta fachada, a la vez obligarían a alejar las posiciones de los atacantes o sitiadores.

El espacio situado entre la falsabraga y los muros del castillo de Don Juan Manuel, denominado liza, no estaría comunicados con la fortaleza a nivel de cota \pm 0 de la liza. Sí que lo estaría por los adarves. Del adarve que delimita el patio de la fortaleza preexistente por su lado este, se pasaría al adarve de la falsabraga, y desde esté se bajaría a la liza.

Es decir la liza y la fortaleza tendrían una comunicación muy difícil, hecho que es muy positivo para la defensa del castillo, por las teorías de la compartimentación defensiva, ya que de esta manera existen más "barreras" para el posible asalto de la fortaleza por esta fachada, pero poco funcional para hacer llegar hasta la liza elementos pesados de grandes dimensiones como las piezas de artillería, la pólvora o los bolardos que se utilizaban como munición, de ahí que fuera necesaria la realización de un acceso independiente de grandes dimensiones como el que aparece en la fachada norte que da acceso directo a la liza, por el que podrían acceder carros o reatas de mulas.

Esta sería la justificación de la necesidad de la existencia de dos puertas en el castillo de Almansa: por cada una de ellas se accedería a un recinto distinto.

Este acceso seguirá siendo un punto débil de la fortificación, por lo cual se dispondrán ciertos mecanismos defensivos en la fachada norte destinados a defender este punto débil.

\subsection{Fachada norte}

Esta fachada, orientada al norte al igual que la anterior, orientada al este, se abre al paso natural que se ha denominado el Corredor de Almansa, o lo que es lo mismo, de fácil aproximación al castillo.

Además, como la fachada sur, se emplaza sobre la capa de estratos blandos que en esa fachada hizo necesaria la construcción del foso, con la diferencia que la cota inferior del estrato débil está más alta en el lado norte del Castillo que en el lado sur, siendo ocupada prácticamente en su totalizad por la fortaleza con la reforma que se haría en el siglo XV para adaptarla a las teorías de la fortificación del momento. Por lo que con esta actuación esta fachada tampoco sería susceptible de ser atacada por mina.

La actuación consistirá en la construcción de un refuerzo, adosado al lienzo norte del castillo de Don Juan Manuel, con mucha masa, y por tanto con una gran inercia, para absorber los posibles impactos de la artillería pirobalíatica, materializado con la técnica del tapial, a modo de baluarte que, dirigiéndose hacia el exterior de la fortaleza ocupa la zona de estratos débiles del cerro y, además por su geometría en planta, en forma de baluarte, sirve para proteger la entrada norte del castillo.

\subsection{Fachada oeste}

La intervención en esta fachada tiene un sentido distinto al que tienen las actuaciones en las otras.

Por su orografía y su naturaleza geológica, el castillo por esta fachada no necesita ninguna actuación para su adaptación defensiva a los nuevos tiempos, ya que está situada en la parte más alta del cerro, junto al mayor desnivel entre el éste y su entorno.

Se trata de un lienzo de muralla natural de grandes dimensiones, por supuesto inmune también al ataque por zapa o mina.

Pero en esta fachada está la torre del homenaje del castillo (Simón y García, 2006), que es la actuación del siglo XV.

En su interior, tanto su espacio principal, que a su vez será el principal del castillo por 
representativo y mejor defendido, como la escalera de caracol (Martínez, 2015) situada en el rincón suroeste de la torre, nos identifican también a esta actuación como una obra del siglo XV.

Desechada la motivación del refuerzo defensivo de la fortaleza para la ejecución de estas actuaciones, para encontrar el por qué de ellas hay que buscarlo en el otro aspecto que caracterizaba las actuaciones de los señores feudales del siglo $\mathrm{XV}$ que no sería otro que "poner de manifiesto el poder del Señor y de impresionar a propios y extraños" (Villena, 2000).

Así es, situada en la cara oeste del cerro, el punto más visible desde la población, es el recuerdo constante para los pobladores de Almansa para que no se olviden quien es su señor: Don Juan Pacheco.

Las actuaciones comenzarían por desmochar la antigua torre almohade que hasta esos momento habría actuado de último reducto defensivo del castillo, conservando el aljibe, que seguirá siendo fundamental para funcionamiento de la nueva torre del homenaje como último reducto defensivo de la fortaleza.

Esta nueva torre, mucho más grande que la preexistente se implanta aproximadamente en el centro de la fachada oeste, jerarquizando la imagen del castillo en esta orientación, aunque esta jerarquía se extiende a todas las vistas debido al gran volumen del elemento en la zona más alta del castillo, visible desde cualquier vista. Pero no se sitúa en el centro geométrico de la fachada, ya que su situación estará condicionada por las preexistencias de los distintos elementos funcionales como el acceso desde el interior del castillo o el aljibe.

Desde la torre, con su posición aproximadamente centrada, se controla todo el castillo, toda la población, todo el paso de los caminos. En definitiva es la expresión del control de todo, y por tanto es la expresión del poder de su propietario.

Se plantean dos accesos a la nueva torre, ambos influenciados por el concepto de la compartimentación de la defensa: el principal en la fachada este de la torre y otro secundario en la fachada norte.

La torre presenta un hueco en cada fachada, pero solo estos dos serían accesos, ya que en función del concepto de la compartimentación defensiva, presentarán barreras para dificultar el acceso a la torre, y/o elementos para incrementar su defensa, mientras que en los otros no se reconocen elementos de este tipo, indicando así el sentido de los recorridos del castillo.

De esta manera el hueco de la fachada oeste sería una ventana, ya que da al exterior del castillo, al acantilado inaccesible por la orografía del cerro, por lo que no necesita ningún elemento para su defesa, y el hueco de la fachada sur, se comportaría como una salida a la parte exterior de la torre, por su orografía y posición en función de los recorridos del castillo, la zona más inaccesible, relacionado con la torre del homenaje, de ahí la ubicación del aljibe en la parte posterior de la torre, según el sentido de los recorridos. Sería para el uso de la torre del homenaje.

La puerta este sería la principal, por eso se presenta enriquecida con un arco conopial a modo de dintel, a la que se accedería desde las construcciones hoy desaparecidas a través de un puente de madera apoyado entre ambas construcciones, que se podría fácilmente desmontar o quemar en caso de peligro para los ocupantes de la torre quedando está aislada del resto del edificio por este acceso.

El acceso norte está concebido como un "acceso elevado", para dificultar su asalto.

Además estaba defendido por un matacán del que aun se pueden contemplar los restos en alguna fotografía antigua, y que las restauraciones del pasado siglo eliminaron.

La puerta de la fachada sur, por su posición en relación con el nivel exterior de la torre, también podría presentarse como un "acceso elevado", pero sobre esta no se aprecian restos de matacán para su defensa, y dado que para llegar a esta puerta solo se puede llegar atravesando la torre del homenaje, es por lo que se considera salida al último recinto del castillo que sería lo que se denomina como ala sur. No sería una entrada. 
Así pues la posición de la torre del homenaje que construye Don Juan Pacheco, está condicionada por el aljibe en su parte sur, y por el norte por las construcciones preexistentes, a través de las cuales se realizará el acceso.

De esta manera, por un lado, el aljibe quedará en la retaguardia de la torre, para su uso en el caso de que ésta quede aislada del resto del castillo y, por otro, las construcciones preexistentes, con la nueva torre, en planta se solapan lo mínimo para que la nueva torre sea lo más inaccesible posible desde éstas.

Es por esto por lo que el acceso principal a la torre del homenaje en la fachada este, se sitúa desplazado hacia el norte.

\section{Conclusiones}

El modelo de fortificación almohade ha sido transformado por el castillo que habitó Don Juan Manuel, y este ha sido transformado por las actuaciones de los Pacheco en el siglo XV. Actuaciones posteriores que enmascaran a las anteriores, por lo que su recuperación conceptual no podrá pasar de la mera hipótesis.

No ocurre lo mismo con el castillo de los Pacheco, con el que se concluye todo un proceso constructivo, cuyo resultado final es un edificio que recoge las teorías de la fortificación del siglo $\mathrm{XV}$, convirtiéndolo en un ejemplo de actuación en una fortificación de este momento.

A partir del conocimiento de su emplazamiento y de su entorno inmediato, se consigue un mecanismo defensivo irrepetible.

Es la respuesta de un lugar a las necesidades de la sociedad del siglo $\mathrm{XV}$, por un lado a las necesidades bélicas y por otro, a la necesidad de la manifestación de poder que la sociedad feudal del momento requería.

Así el castillo de los Pacheco del siglo XV es el único modelo de edificio completo del castillo de Almansa que se puede reconocer, puesto que en él se incorpora todo lo construído hasta ese momento, ya sea de nueva planta o modificado sobre construcciones anteriores, y a partir de él no se realizan más intervenciones, al menos de envergadura, puesto que a partir del siglo XVI los castillos dejan de tener el valor como fortificaciones, y por lo tanto, a no ser que se les encuentre otro uso, son abandonados.

Dos son los aspectos más importantes que caracterizan el modelo del castillo del siglo $\mathrm{XV}$ : la antropización del cerro adaptándolo a la función defensiva de la fortaleza y el concepto de la compartimentación de la defensa.

El primero hace único al castillo de Almansa.

El segundo da sentido al edificio.

El concepto de la compartimentación de la defensa es el que organiza el edificio del castillo de Almansa como mecanismo defensivo ejemplar, disponiendo la serie de obstáculos y defensas, compartimentando diferentes recintos en el castillo de forma tal que se potencie una defensa escalonada, de forma que la pérdida de una parte no comprometa la defensa de las siguientes, poniendo obstáculos para su progresión interior, y que exista un último reducto (torre del Homenaje) capaz de servir de refugio frente a la pérdida del resto de la fortaleza.

Así el castillo de Almansa tendría los siguientes sectores, que caído uno, no compromete al siguiente.

1.- Torre albarrana (Torre T5)

2.- Barbacana

3.- Foso

4.- Liza y falsabraga artillada

5.- Patio

6.- Torre y edificio de arcos diafragmáticos

7.- Torre del Homenaje y ala sur.

De esta manera, el castillo de Almansa constructiva y funcionalmente se comporta como un ejemplo de las teorías de la fortificación del siglo XV. 


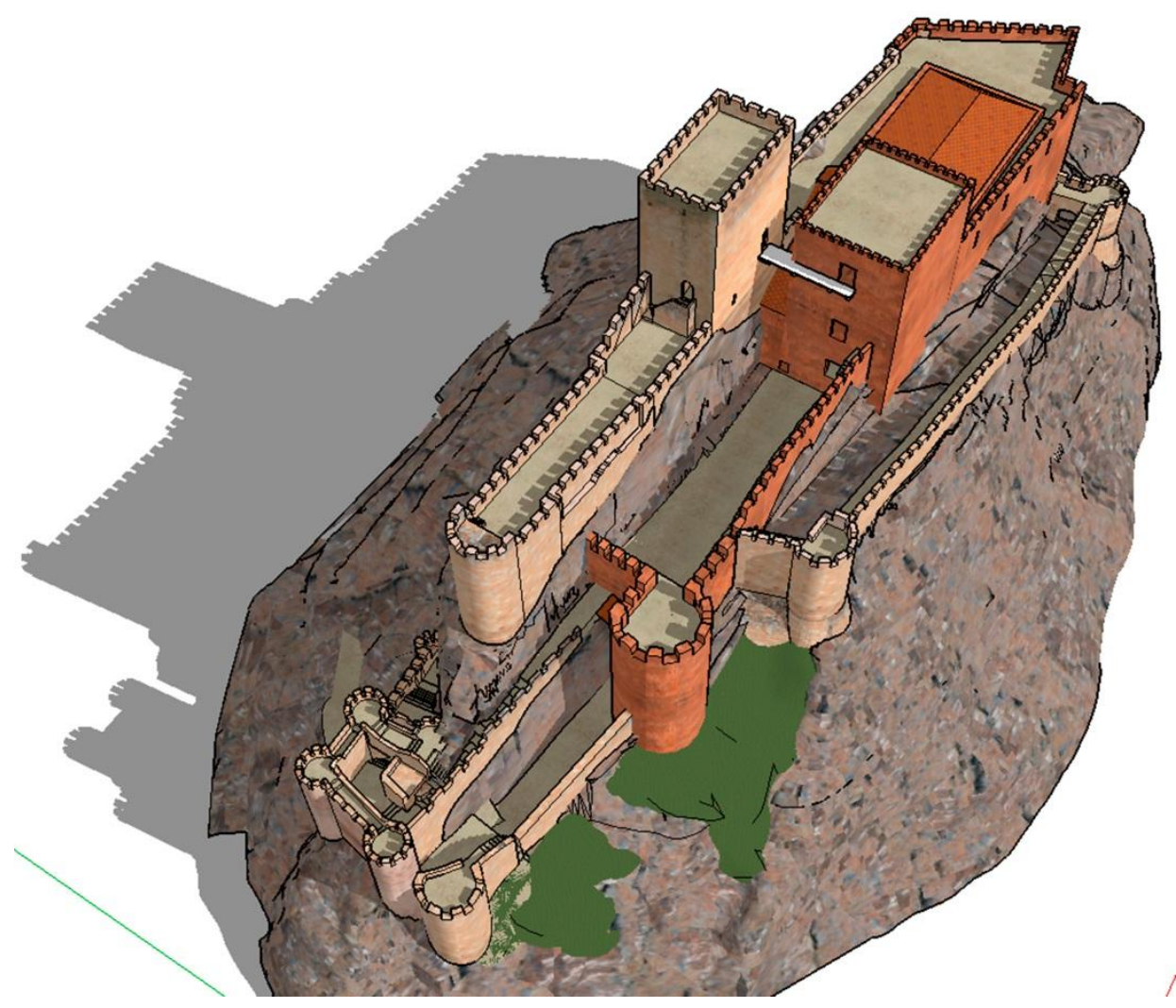

Fig. 3- Hipótesis del Castillo del siglo XV (Joaquín Fco. García, 2015)

\section{Referencias}

De Mora-Figueroa, L. (2006). Glosario de arquitectura defensiva medieval. Ed. Ministerio de Defensa. Madrid. pp. 105-107, 196.

García Sáez, J. F. (2011) "La construcción de un castillo" en Actas del VII Congreso Nacional de Historia de la construcción. Ed. Instituto Juan de Herrera. Santiago de Compostela. pp. 527-537.

García Sáez, J. F. (2015) “El castillo que no vemos. Reflexiones acerca del Castillo de Almansa”, en Jornadas de estudios locales $n^{\circ} 11$ "El Castillo de Almansa: Un símbolo del pasado con proyección de futuro". Ed. Excmo. Ayuntamiento de Almansa. En imprenta.

Gil Hernández, E. R. (2008) MEMORIA SEGUIMIENTO ARQUEOLÓGICO del Proyecto de Restauración en el Elemento Lienzo T1/T10 Exterior (paño de tapial) del Castillo de Almansa (Albacete). Inédito. Promotor: Ayuntamiento de Almansa.

Martínez García, O. J. (2015) Arquitectura gótica y barroca en Almansa. Nuevas aportaciones. Ed. I.E.A. "Don Juan Manuel”. Albacete. pp. 25-63.

Simón García, J. L. y García Sáez, J. F. "Arquitectura gótica en Almansa: Testigos de una época épica”. en Jornadas de estudios locales $n^{\circ} 6$ "Arquitectura religiosa en Almansa". Ed. Excmo. Ayuntamiento de Almansa. Almansa 2006. pp. 45-72.

Uriel Ortiz, Á. y Puebla Contreras F. J. 1.990. Reparación y estabilización de los agrietamientos del Castillo de Almansa. Ed. URIEL\&ASOCIADOS. Pozuelo de Alarcón.

Villena, L. (1998) “CCómo eran los Castillos Medievales?” en Actas del I Congreso de Castellología Ibérica. Ed. Asociación Española de Amigos de los Castillos. Palencia. pp. 60-63. 